\title{
UN HÍBRIDO ESPONTÁNEO ENTRE ARISTOLOCHIA GORGONA Y A. GRANDIFLORA (ARISTOLOCHIACEAE)
}

\author{
Mario A. Blanco \\ Department of Botany, University of Florida, 220 Bartram Hall, Gainesville, Florida 32611-8526, USA; Jardín \\ Botánico Lankester, Universidad de Costa Rica, 1031-7050 Cartago, Costa Rica; e Instituto Centroamericano para la \\ Investigación Biológica y la Conservación, 2398-250 San José, Costa Rica. mblanco@flmnh.ufl.edu
}

\begin{abstract}
A spontaneous hybrid between Aristolochia gorgona and A. grandiflora appeared at Lankester Botanical Garden, Costa Rica, where plants of both species were cultivated in close proximity. This indicates that geographic isolation maintains the genetic integrity of these two closely related species. The flower morphology of $A$. grandiflora is dominant in the $\mathrm{F}_{1}$ hybrids. The few known cases of hybridization in the genus are listed.
\end{abstract}

RESUMEN. Un híbrido espontáneo entre Aristolochia gorgona y A. grandiflora apareció en el Jardín Botánico Lankester, Costa Rica, donde plantas de ambas especies fueron cultivadas próximas unas a otras. Esto indica que el aislamiento geográfico mantiene la integridad genética de estas dos especies cercanamente emparentadas. La morfología floral de $A$. grandiflora es dominante en los híbridos $\mathrm{F}_{1}$. Se enumeran los pocos casos conocidos de hibridación en el género.

PALABRAS CLAVE / KeY wORDS: Aristolochiaceae, Aristolochia, hibridación espontánea, spontaneous hybridization, Costa Rica.

Aristolochia gorgona M.A. Blanco es una especie de flores muy grandes, descrita recientemente de Costa Rica y Panamá (Blanco 2002). Está estrechamente emparentada con A. grandiflora Sw. (Figs. 1 y 2), la cual está distribuida naturalmente desde el sur de México hasta el occidente de Ecuador y en algunas islas del Caribe; además, es cultivada en muchas partes del mundo como ornamental. Como muchas especies de Aristolochia, A. grandiflora y A. gorgona son plantas de elevaciones bajas. En Costa Rica, y al menos en la mitad oeste de Panamá, ambas especies están aisladas geográficamente por cadenas montañosas: A. grandiflora está restringida a la vertiente pacífica, mientras que $A$. gorgona está restringida a la vertiente caribeña. Fotografías adicionales de las flores en vida de ambas especies están disponibles en internet en W3TROPICOS (http://mobot.mobot.org/W3T/Search/ vast.html).

En enero de 2001, cortes de tallo de Aristolochia gorgona provenientes de la localidad típica se cultivaron en el Jardín Botánico Lankester, Cartago, Costa Rica. Uno de éstos se sembró a pocos metros de una planta adulta de $A$. grandiflora y creció rápidamente. Poco más de un año después empezó a florecer, y en junio de 2002 produjo dos frutos. Las semillas fueron distribuidas entre algunos aficionados al cultivo de estas plantas (ver sitio de internet en http://groups.
yahoo.com/group/Aristolochia/) en Australia, Brasil, Estados Unidos de América, Francia e Italia, con la suposición de que eran producto de autopolinización. También se enviaron semillas al Jardín Botánico de Missouri en EE.UU. y al Jardín Botánico de Múnich en Alemania. Algunas personas que recibieron semillas lograron germinarlas y las plántulas crecieron rápidamente, y empezaron a florecer aproximadamente un año después en al menos tres países distintos. Las fotografías enviadas por estas personas demuestran que las plantas corresponden a un cruce espontáneo entre A. gorgona y A. grandiflora (Figs. 3-4). En el año 2004 también florecieron plantas híbridas en el Jardín Botánico Lankester y en la Universidad de Florida. Los siguientes ejemplares testigo se depositaron en el Herbario de la Universidad de Costa Rica (USJ): $A$. gorgona (Blanco 2666), A. grandiflora (Blanco 2665), y A. gorgona x A. grandiflora (Blanco 2664). También del híbrido, se depositaron los siguientes ejemplares en el Herbario de la Universidad de Florida (FLAS): Bliss 04.005a, Bliss 04.005b, y Blanco 2687.

El término "híbrido espontáneo" se utiliza aquí para designar un cruce interespecífico de plantas cultivadas fuera de su hábitat natural, pero no producto directo de la intervención humana (esto es, por polinización cruzada "accidental"). Por lo tanto, los términos "híbrido natural" e "híbrido artificial", en su sentido 
tradicional, son inapropiados en este caso.

La pronta aparición de un híbrido en condiciones de cultivo entre estas especies indica que ambas utilizan el mismo tipo de dípteros polinizadores, y que el aislamiento geográfico es el factor responsable de mantener su integridad genética. Es posible que haya áreas de simpatria en el este de Panamá (he estudiado ejemplares de herbario de ambas especies recolectadas en diferentes sitios de la provincia de Darién). Si este híbrido existiera en forma natural, sería muy difícil distinguirlo de Aristolochia grandiflora en ejemplares secos de herbario. Incluso muchas muestras de $A$. gorgona pasaron largo tiempo determinadas como A. grandiflora antes de que sus flores en vida fueran estudiadas y descritas (Blanco 2002).

Es bien conocido que Aristolochia grandiflora es polinizada por una variedad de dípteros de diferentes familias en diferentes partes del mundo, que son atraídos a las flores por su fuerte olor a carne en descomposición (Burgess et al. 2004, Cammerloher 1923, Hart 1897, Hilje 1984, Hubbard 1877a,b, Petch 1924). A pesar de las diferencias en la configuración del limbo y en la estructura de los osmóforos (Blanco 2002), ambas especies emiten un olor semejante, y el utrículo, aunque más grande en $A$. gorgona, tiene una forma parecida. Brantjes (1980) encontró en Brasil que las diferencias en el diámetro del tubo del cáliz ("cuello de botella") y la distancia entre el ginostemio y la pared adyacente del utrículo en especies simpátricas determinan diferentes especies de dípteros polinizadores; sin embargo, no parece haber diferencias sustanciales en cuanto a estas características entre $A$. gorgona y $A$. grandiflora. Petch (1924) observó que diferentes especies de Aristolochia cultivadas en Sri Lanka atraían grupos diferentes de dípteros (algunas especies se compartían y otras no); no obstante, las flores de $A$. gorgona y $A$. grandiflora en el Jardín Botánico Lankester atraen básicamente al mismo conjunto de dípteros (observación personal).

Las flores del híbrido $\mathrm{F}_{1}$ comparten características de ambas especies, pero el fenotipo de Aristolochia grandiflora es claramente dominante: el apéndice floral es casi tan largo como en A. grandiflora, y el patrón de manchas es muy parecido al de esta especie (Figs. 3 y 4). La influencia de A. gorgona es más sutil, y se expresa en el color de fondo amarillento del limbo (A. grandiflora tiene un color base blanco sucio), y en la presencia de pequeños tubérculos o verrugas oscuras alrededor de la boca del tubo floral (Fig. 6), vestigios de los osmóforos tentaculoides de
A. gorgona (Fig. 5). Además el limbo del híbrido no es cóncavo como en A. grandiflora, sino que la parte distal se curva ligeramente hacia atrás, y los márgenes tienden a plegarse hacia la parte frontal, como en $A$. gorgona (Fig. 4).

Ambas especies son muy parecidas vegetativamente, pero Aristolochia grandiflora tiene hojas algo alargadas con lóbulos basales erectos. Por su parte, A. gorgona tiene hojas más redondeadas con lóbulos basales curvados hacia adentro. Las hojas del híbrido son intermedias en forma, pues son alargadas con lóbulos basales ligeramente curvados hacia adentro (Fig. 3). Blanco (2002) presenta una enumeración más detallada de las diferencias entre $A$. grandiflora y $A$. gorgona.

Hay pocos informes de hibridación en Aristolochia. El más conocido es un cruce de A. labiata Willd. (sinónimo: A. brasiliensis Mart. \& Zucc.) con A. trilobata L. (sinónimo: A. macroura Gómez), realizado primero artificialmente por Ule (1899) en Brasil. El mismo híbrido apareció años más tarde en forma espontánea en los Reales Jardines Botánicos de Kew, Inglaterra, donde fue bautizado con el nombre informal (inválidamente publicado) "A. kewensis" (Watson 1911, Petch 1924); posteriormente, también apareció en República Dominicana (Schmidt 1935: 393-394), de donde fue descrito formalmente como A. domingensis Ekman \& Schmidt [originalmente como especie, pero corresponde al mismo híbrido según Pfeifer (1966: 157); tipo: Ekman 14520 (S!)]. Este y otros tres cruces artificiales, $A$. gigantea Mart. x A. ringens Vahl, $A$. gigantea x A. labiata y A. elegans Mast. x A. labiata, fueron documentados con fotografías en el foro de internet arriba mencionado (mensaje No. 782, 22 agosto 2002). Ule (1899: 36) también realizó el último cruce mencionado, pero todos los frutos abortaron; sin embargo, cruzó con éxito $A$. trilobata con A. elegans (Ule 1899: 38); a pesar de que obtuvo plántulas y manifestó su intención de describir el híbrido una vez que floreciera, nunca llegó a hacerlo. Aristolochia compta Hoehne fue descrita como un posible híbrido espontáneo entre $A$. brasiliensis $(=A$. labiata) y $A$. triangularis Cham. (Hoehne 1947); apareció en el Jardín Botánico de São Paulo, Brasil, entre plantas cultivadas de los supuestos progenitores. No conozco ningún caso confirmado de híbridos naturales, pero es muy posible que existan en un género tan grande.

Es notable que a pesar de la amplia disponibilidad de Aristolochia grandiflora en cultivo desde hace 
muchas décadas, y de lo llamativo de sus flores, no existen registros previos de su uso en hibridación. Posiblemente se ha intentado cruzarla en el pasado, pero los resultados negativos en hibridación pocas veces son publicados. Es posible que los cruces de A. grandiflora con la mayoría de las especies de Aristolochia sean incompatibles, debido a que está lejanamente emparentada con otras especies del género, de acuerdo al análisis filogenético de Neinhuis et al. (2004), el cual no incluyó a $A$. gorgona. Análisis moleculares adicionales indican que $A$. grandiflora, $A$. gorgona, y A. pichinchensis Pfeifer (aquí considerada como especie distinta de $A$. grandiflora) constituyen un grupo bien definido, relativamente aislado dentro del género (Neinhuis \& Wanke, datos no publicados).

Agradecimientos. A Juan Carlos Cervantes Moya, por su dedicación al cultivo de la colección de Aristolochia en el Jardín Botánico Lankester, y a Emily Serrano por su asistencia con las fotografías. Barbara Bliss (Pennsylvania State University), Günter Gerlach (Botanischer Garten MünchenNymphenburg), Mary Merello (Missouri Botanical Garden), Enid Offolter (Natural Selections Exotics, Fort Lauderdale, Florida), y Mauro Peixoto (São Paulo, Brasil) germinaron y cultivaron los híbridos hasta su floración, me enviaron fotografías de sus flores, y generosamente me permitieron utilizarlas en esta nota. Mia Ehn Salter (S) gentilmente me envió imágenes del tipo de A. domingensis. Christoph Neinhuis y Stefan Wanke (Institut für Botanik, Dresden) amablemente compartieron resultados no publicados de sus análisis filogenéticos. Los comentarios de dos revisores anónimos ayudaron a mejorar el manuscrito.

\section{LITERATURA CITADA}

Blanco, M.A. 2002. Aristolochia gorgona, a new species with giant flowers from Costa Rica and Panama. Brittonia 54: 30-39.

Brantjes, N.B.M. 1980. Flower morphology of Aristolochia species and the consequences for pollination. Acta Bot. Neerl. 29: 212-213.

Burgess, K.S., J. Singfield, V. Meléndez, \& P.G. Kevan. 2004. Pollination biology of Aristolochia grandiflora (Aristolochiaceae) in Veracruz, Mexico. Ann. Missouri Bot. Gard. 91: 346-356.

Cammerloher, H. 1923. Zur Biologie der Blüte von Aristolochia grandiflora Swartz. Österr. Bot. Zeitschr. 72: 180-198.

Hart, J.H. 1897. Necrophagous diptera. Bull. Misc. Inform. Roy. Bot. Gard. Trinidad 3: 28-29.

Hilje, L. 1984. Fenología y ecología floral de Aristolochia grandiflora Swartz (Aristolochiaceae) en Costa Rica. Brenesia 22: 1-44.

Hoehne, F.C. 1947. Novas espécies e contribuções para o conhecimento do gênero Aristolochia na América do Sul. Arq. Bot. Estado São Paulo n.s., formato maior 2: 95-103 (+ Tabs. 32-39).

Hubbard, H.G. 1877a. Cross-fertilization of Aristolochia. Amer. Nat. 11: 303-304.

Hubbard, H.G. 1877b. Cross-fertilization of Aristolochia. Bot. Gaz. 2: 121-122.

Neinhuis, C., S. Wanke, K.W. Hilu, K. Müller \& T. Borsch. 2004. Phylogeny of Aristolochiaceae based on parsimony, likelihood, and Bayesian analyses of $\operatorname{trn} \mathrm{L}-t r n \mathrm{~F}$ sequences. P1. Syst. Evol. 250: 7-26.

Petch, T. 1924. Notes on Aristolochia. Ann. Roy. Bot. Gard. (Peradeniya) 8: 1-108.

Pfeifer, H.W. 1966. Revision of the North and Central American hexandrous species of Aristolochia (Aristolochiaceae). Ann. Missouri Bot. Gard. 53: 1-114.

Schmidt, O.C. 1935. Beiträge zur Kenntnis der Aristolochiaceen V. Notizbl. Bot. Gart. BerlinDahlem 12: 389-394.

Ule, E. 1899. Ueber einen experimentel erzeugten Aristolochienbastard. Ber. Deutsch. Bot. Ges. 17: 35-40.

Watson, W. 1911. New or noteworthy plants: Aristolochia kewensis (A. trilobata x A. brasiliensis). Gard. Chron. 50: 300-301. 


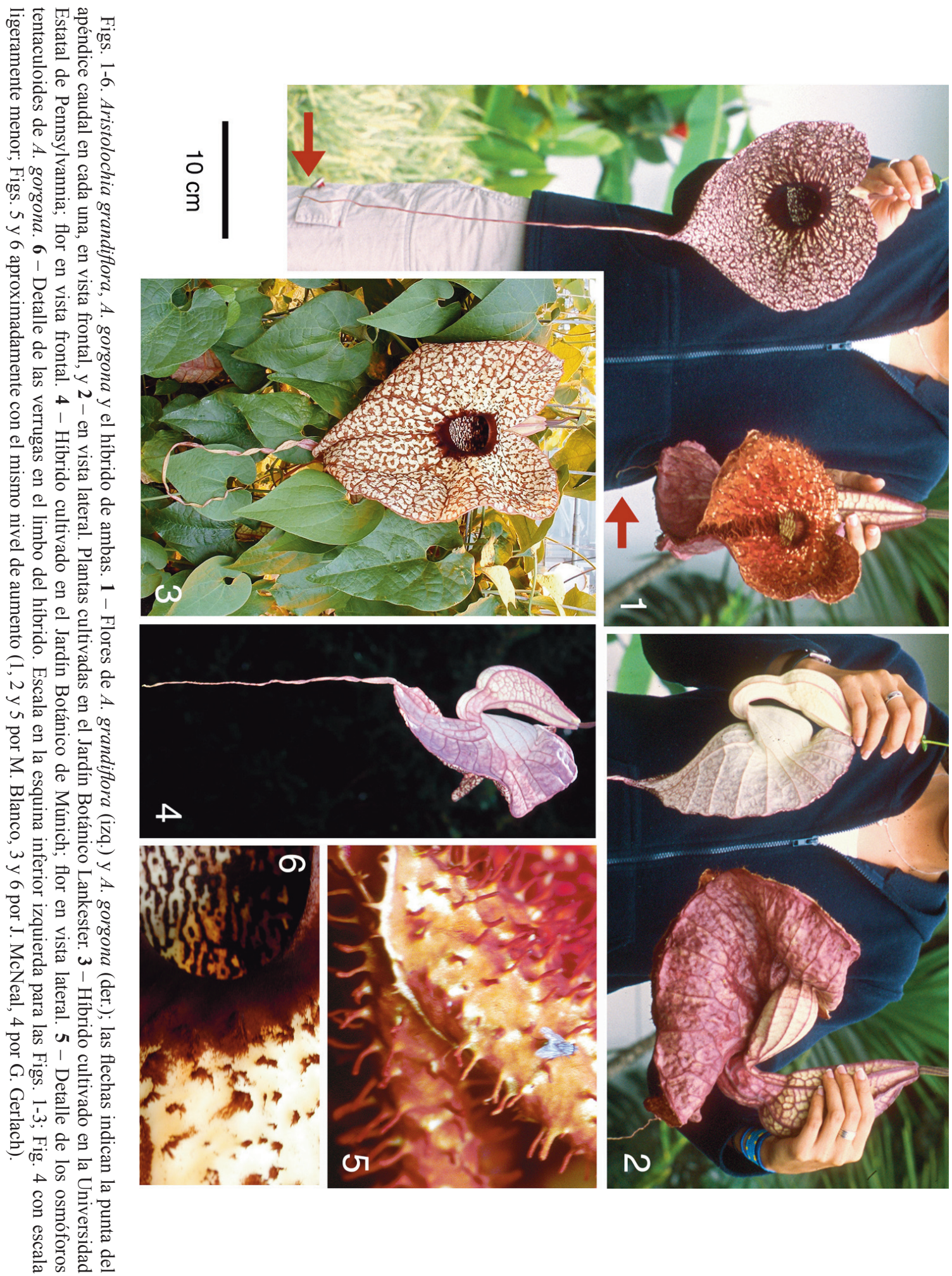

